\title{
NOTA
}

\section{NETEROSIVIDADE MG: EROSIVIDADE DA CHUVA EM MINAS GERAIS ${ }^{(1)}$}

\author{
Michel Castro Moreira ${ }^{(2)}$, Fernando Falco Pruski ${ }^{(3)}$, Thiago Emanuel \\ Cunha de Oliveira ${ }^{(4)}$, Francisco de Assis de Carvalho Pinto ${ }^{(5)} \&$ \\ Demetrius David da Silva ${ }^{(5)}$
}

\begin{abstract}
RESUMO
A erosividade da chuva é um índice numérico que expressa a capacidade das chuvas em provocar erosão hídrica no solo. O presente trabalho teve por objetivo desenvolver um programa computacional para estimar os valores da erosividade da chuva no Estado de Minas Gerais com base em redes neurais artificiais (RNAs). O valor anual da erosividade da chuva é obtido pelo somatório dos valores mensais dos índices de erosividade $\mathrm{EI}_{30}$ ou $\mathrm{KE}>25$. Foram utilizados para cálculo de cada um desses índices dois métodos de obtenção da energia cinética de precipitação pluvial. Dessa maneira, obtiveram-se quatro valores de erosividade para cada mês, totalizando o desenvolvimento de 48 redes. As RNAs desenvolvidas foram implementadas no ambiente de programação Borland Delphi 7.0. O programa computacional desenvolvido foi denominado NetErosividade MG. O programa fornece, de forma fácil e rápida, os valores mensais e anual da erosividade da chuva para qualquer localidade do Estado de Minas Gerais.
\end{abstract}

Termos de indexação: redes neurais artificiais, conservação do solo, equação universal de perda de solo.

SUMMARY: NETEROSIVIDADE MG: RAINFALL EROSIVITY FOR MINAS GERAIS STATE, BRAZIL

Rainfall erosivity represents the potential of rainfall causing soil erosion. This study aimed to develop a software to estimate rainfall erosivity in the state of Minas Gerais

\footnotetext{
(1) Recebido para publicação em junho de 2007 e aprovado em março de 2008.

${ }^{(2)}$ Doutorando em Engenharia Agrícola, Departamento de Engenharia Agrícola, Universidade Federal de Viçosa - UFV. Av. P.H. Rolfs s/n, CEP 36570-000 Viçosa (MG). Bolsista do CNPq. E-mail: mmoreira@gprh.ufv.br

(3) Professor Titular, Departamento de Engenharia Agrícola, UFV. Bolsista do CNPq. E-mail: ffpruski@ufv.br

(4) Graduando em Ciência da Computação, Departamento de Informática, UFV. Bolsista da FAPEMIG. E-mail: thiagoemanuel@yahoo.com.br

(5) Professor Adjunto, Departamento de Engenharia Agrícola, UFV. Bolsista do CNPq. E-mail: facpinto@ufv.br; david@ufv.br
} 


\begin{abstract}
based on Artificial Neural Networks (ANNs). The annual value of the rainfall erosivity is given by the sum of the monthly values of the erosivity indexes $E I_{30}$ or $K E>25$. Two methodologies were used to estimate the kinetic energy for each index. Thus, four erosivity values were evaluated for each month, resulting in the development of 48 ANNs. These ANNs were implemented using the software Borland Delphi 7.0. The new software was called NetErosividade MG. The program calculates the monthly and annual values of rainfall erosivity for any location in the state of Minas Gerais in an easy and fast way.
\end{abstract}

Index terms: artificial neural networks, soil conservation, universal soil loss equation.

\section{INTRODUÇÃO}

A erosão consiste no processo de desprendimento e transporte das partículas do solo, sendo responsável por grandes prejuízos, não só para a exploração agropecuária, mas também para diversas atividades econômicas e para o próprio meio ambiente (Amorim, 2004).

Wischmeier \& Smith (1958) desenvolveram a Equação Universal de Perda de Solo (USLE), que permite estimar as perdas de solo e identificar os fatores que exercem o maior efeito sobre estas. Dentre os fatores da USLE, o que expressa a capacidade erosiva da chuva é conhecido como fator erosividade da chuva (R).

A determinação da erosividade da chuva envolve um trabalho exaustivo de seleção e leitura de uma série de registros de chuvas, sendo esses dados escassos e, muitas vezes, de difícil acesso (Mello et al., 2007). Tal fato faz com que informações relativas à erosividade estejam disponíveis apenas para pequeno número de localidades no Brasil (Moreira et al., 2006b).

Dada a dificuldade em obter o valor experimental da erosividade da chuva, diversos autores, como Bertoni \& Lombardi Neto (1999), Lombardi Neto et al. (1999), Silva (2004) e Moreira et al. (2006b), têm utilizado técnicas de interpolação para a estimativa dos valores de $R$.

De acordo com Sárközy (1999), as redes neurais artificiais (RNAs) podem ser usadas como ferramentas de interpolação. Uma RNA é uma estrutura computacional criada para imitar o funcionamento do cérebro humano, adquirindo conhecimento por intermédio do processo de treinamento, a fim de encontrar pesos para as diferentes conexões (parâmetros livres W's e b's) entre os elementos processadores da rede, denominados neurônios artificiais (Persson et al., 2002).

Moreira et al. (2007) desenvolveram RNAs para a estimativa dos valores mensais de $\mathrm{R}$ para qualquer localidade do Estado de Minas Gerais. Na avaliação das RNAs desenvolvidas, os autores utilizaram, além do coeficiente de determinação $\left(\mathrm{R}^{2}\right)$, o índice de confiança (c), proposto por Camargo \& Sentelhas (1997), o qual é calculado pelo produto do coeficiente de correlação (r) e do índice de concordância (d), proposto por Willmott (1981) e concluíram que as RNAs desenvolvidas possibilitam estimar a erosividade mensal da chuva, constituindo alternativa viável para a interpolação desses valores no Estado de Minas Gerais.

As RNAs desenvolvidas, no entanto, necessitam, para sua utilização, de um programa computacional proprietário, de alto custo, além de adequado treinamento para seu uso. Estes fatos dificultam o seu uso por técnicos e extensionistas, os usuários que mais necessitam de ferramentas práticas que auxiliam na conservação de água e solo (Moreira et al., 2006a).

Dessa forma, a fim de difundir as RNAs e permitir a sua utilização por técnicos e extensionistas, o presente trabalho teve por objetivo desenvolver um programa computacional que viabilize o uso de RNAs para estimativa dos valores da erosividade da chuva no Estado de Minas Gerais.

\section{MATERIAL E MÉTODOS}

Considerando que o valor anual da erosividade da chuva é obtido pelo somatório dos valores mensais dos índices de erosividade $\mathrm{EI}_{30}$ ou $\mathrm{KE}>25$, e que, para o cálculo desses índices, existem dois métodos de obtenção da energia cinética de precipitação pluvial (Foster et al., 1981; Wagner \& Massambani, 1988), Moreira et al. (2007) desenvolveram quatro RNAs para cada mês, permitindo assim a estimativa mensal da erosividade da chuva para qualquer localidade do Estado de Minas Gerais.

Os valores de erosividade da chuva utilizados para o desenvolvimento das RNAs foram obtidos segundo o método proposto por Pruski et al. (2007), em que se estima o valor da erosividade da chuva a partir de séries sintéticas de precipitação pluvial. Para o treinamento das RNAs, foram utilizadas 254 estações pluviométricas situadas no Estado de Minas Gerais e 14 estações localizadas em seu entorno.

De posse das RNAs desenvolvidas, foi necessário conhecer as respectivas arquiteturas, funções de ativação dos neurônios artificiais e os parâmetros livres W's e b's para, então, serem geradas as funções matemáticas que as representem, segundo o modelo apresentado na equação: 


$$
y^{i j}=f\left(\sum_{i=1}^{n} y^{i(j-1)} w_{i^{\prime}}^{i j}+b^{i j}\right)
$$

em que $y^{i j}=$ valor de saída do neurônio $i$ da camada $j$; $\mathrm{n}=$ número de neurônios da camada anterior; $y^{i(j-1)}=$ valor de saída do neurônio $i$ 'da camada anterior; $\mathrm{w}^{i j}$, = valor do peso sináptico do neurônio $i$ da camada $j$, ativado pelo neurônio $i$ ' da camada anterior; $b^{i j}=$ valor de compensação do neurônio $i$ da camada $j$; e $f=$ função de ativação do neurônio $i$.

As RNAs são do tipo retroalimentada e apresentam uma arquitetura $3-\mathrm{n}_{1}-\mathrm{n}_{2}-1$, sendo estes valores correspondentes a um vetor de entrada com três variáveis, duas camadas intermediárias com $\mathrm{n}_{1}$ e $\mathrm{n}_{2}$ neurônios artificiais e um neurônio na camada de saída (Figura 1).

O vetor de entrada é composto pelos valores da latitude e da longitude, em graus decimais, bem como pelo valor de altitude, em metro. No neurônio da camada de saída, tem-se uma função de ativação linear para fornecer o valor da erosividade da chuva da localidade representada pelo vetor de entrada, em MJ mm h-1 ha-1 ano ${ }^{-1}$, para estimativa considerando o $\mathrm{EI}_{30}$, ou em $\mathrm{MJ} \mathrm{ha}^{-1}$ ano $^{-1}$ para estimativa considerando o $\mathrm{KE}>25$. As funções de ativação dos neurônios das camadas intermediárias são do tipo tangente hiperbólica sigmóide. As equações 2 e 3 ilustram as funções de ativação linear e tangente hiperbólica sigmóide (MatLab, 2000).

$$
\operatorname{lin}(x)=x
$$

em que $x=$ valor normalizado, adimensional.

$$
\tanh \operatorname{sig}(x)=\frac{2}{\left(1+e^{-2^{*} x}\right)-1}
$$

Os parâmetros livres w's e b's das RNAs foram exportados em arquivos-texto, nos quais foram armazenadas todas as informações referentes às RNAs. Assim, de posse das arquiteturas, funções de ativação dos neurônios e dos parâmetros livres w's e $b$ 's, foi possível estruturar as 48 equações matemáticas representativas das RNAs e então implementá-las computacionalmente, utilizando-se o ambiente de programação Borland Delphi 7.0.

Considerando que as RNAs desenvolvidas permitem estimar os valores mensais da erosividade da chuva, implementou-se uma rotina que calcula a erosividade anual da chuva a partir dos valores mensais.

Uma interface gráfica foi desenvolvida para que o usuário visualize o mapa do Estado de Minas Gerais e com a simples pressão do mouse sobre o mapa, obtenha os valores mensais e anual da erosividade da chuva para a localidade de interesse. Para tanto, o mapa foi georreferenciado, de modo que, ao pressioná-lo o programa acesse os valores de latitude, longitude e altitude da localidade de interesse, para, então, estimar os valores de $\mathrm{R}$.

No caso de se dispor das coordenadas geográficas e da altitude da localidade em que se deseja conhecer os valores de $R$, uma caixa de grupo foi fornecida para entrada manual desses dados e posterior clique no botão "Calcular".

Uma listagem com os nomes dos municípios do Estado de Minas Gerais foi disposta juntamente com o mapa para permitir ao usuário obter os valores de $R$ pelo nome da localidade de interesse.

Pelo fato de as RNAs necessitarem do valor de altitude para efetuar o cálculo da erosividade da chuva para determinada localidade, foi incorporada ao programa computacional uma base de dados altimétricos, sendo seu acesso feito automaticamente a partir da identificação de uma localidade. As informações de altitude foram obtidas do Projeto

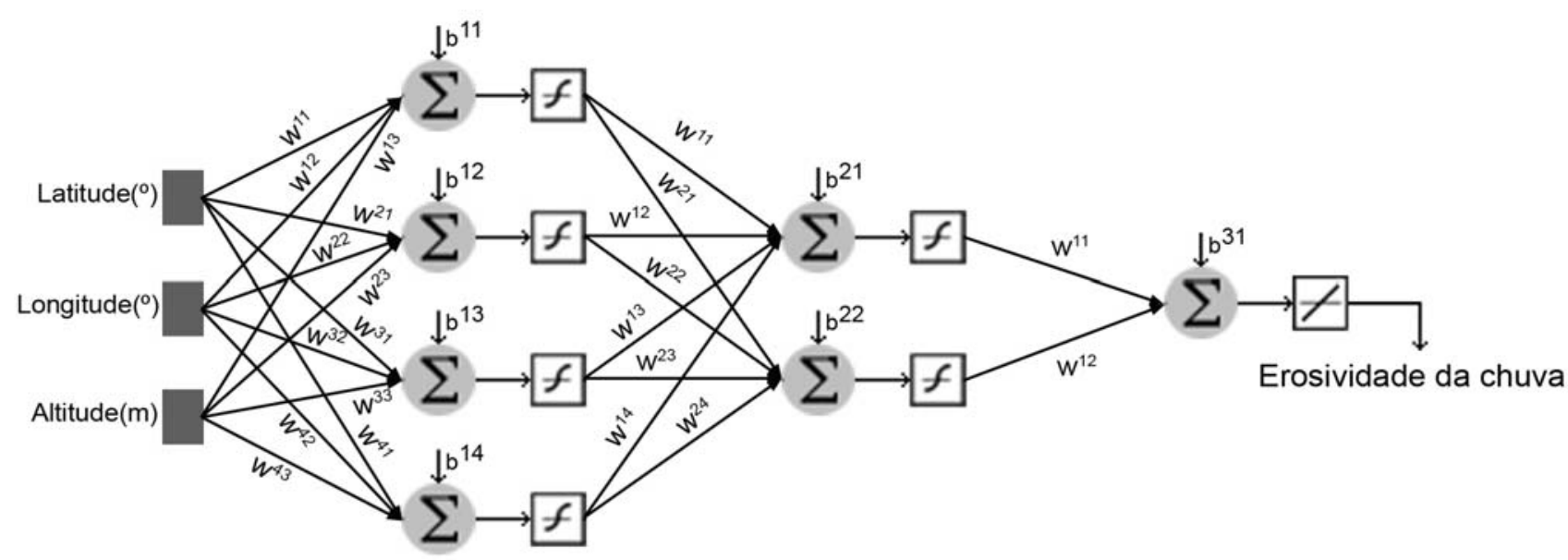

Figura 1. Exemplo de arquitetura 3-4-2-1 de uma rede neural artificial para estimativa da erosividade da chuva. 
GTOPO30, que, segundo Ribeiro et al. (2002), é uma fonte de informação de altimetria que apresenta resolução horizontal de $1 \mathrm{~km}$, tendo sido desenvolvido em escala mundial pelo United States Geological Survey (USGS).

\section{RESULTADOS E DISCUSSÃO}

O programa computacional desenvolvido para estimativa dos valores mensais e anual da erosividade da chuva para qualquer localidade do Estado de Minas Gerais foi denominado NetErosividade $M G$ e pode ser obtido, gratuitamente, no endereço eletrônico http:// www.ufv.br/dea/gprh. A figura 2 ilustra sua tela de apresentação, constando nela seu nome, sua finalidade, a instituição e o grupo responsável pelo seu desenvolvimento.

Na tela principal do NetErosividade $M G$ (Figura 3), o usuário identifica a localidade de interesse, podendo este procedimento ser realizado de três formas: a primeira é a partir do clique do mouse sobre o mapa do Estado de Minas Gerais (campo 1), a segunda é a partir da escolha do nome de um município do Estado (campo 2), e a terceira é a partir do fornecimento dos valores da latitude, longitude e altitude da localidade de interesse (campo 3).

Ao proceder à escolha de uma localidade, são dispostas no mapa duas linhas (horizontal e vertical), indicando, na sua interseção, a localidade selecionada. Os valores de erosividade estimados são dispostos no campo 4 (Figura 3), no qual se observam as abas referentes aos meses do ano e a última (Anual), representando a erosividade da chuva anual. Cabe ressaltar que Moreira et al. (2007) não desenvolveram RNAs referentes à erosividade da chuva anual, sendo os valores anuais apresentados no NetErosividade $M G$ resultado do somatório das erosividades mensais.

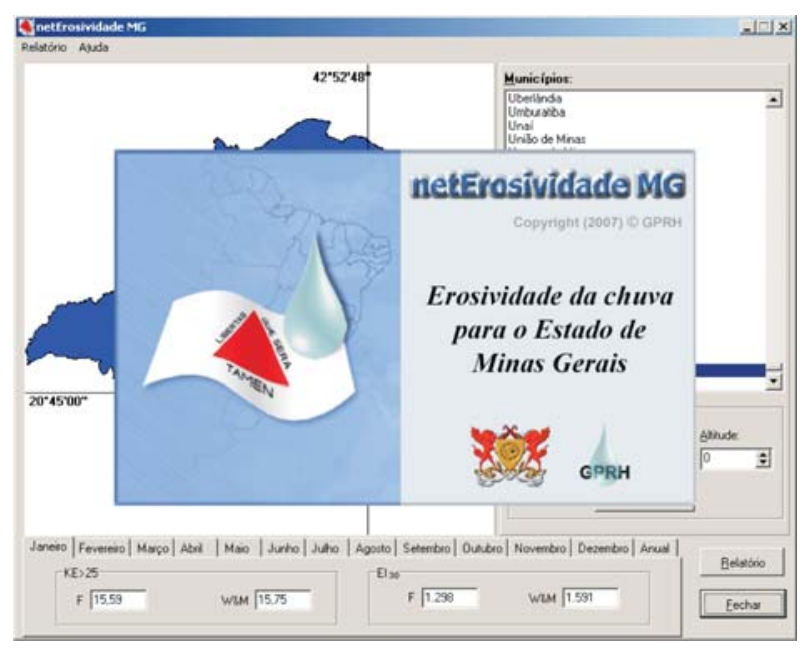

Figura 2. Tela de apresentação do NetErosividade MG.
O NetErosividade $M G$ permite imprimir relatórios com as informações pertinentes à localização (Nome, Latitude, Longitude e Altitude), o mapa do Estado, ilustrando o local de interesse, e os valores mensais e anual da erosividade da chuva, considerando os índices de erosividade $\mathrm{EI}_{30}$ e $\mathrm{KE}>25$ e os dois métodos de obtenção da energia cinética de precipitação pluvial (Foster et al., 1981; Wagner \& Massambani, 1988). Para tanto, basta o usuário pressionar o botão "Relatório" (campo 5). Na figura 4, apresenta-se um relatório emitido pelo NetErosividade $M G$ para a localidade de Viçosa.

Acompanha o NetErosividade $M G$ um sistema de ajuda que permite ao usuário obter informações relativas à utilização do programa computacional e a aspectos teóricos relacionados com as redes neurais artificiais, erosividade da chuva e conservação de solo. Na figura 5, apresenta-se a tela pertinente ao sistema de ajuda do NetErosividade $M G$.

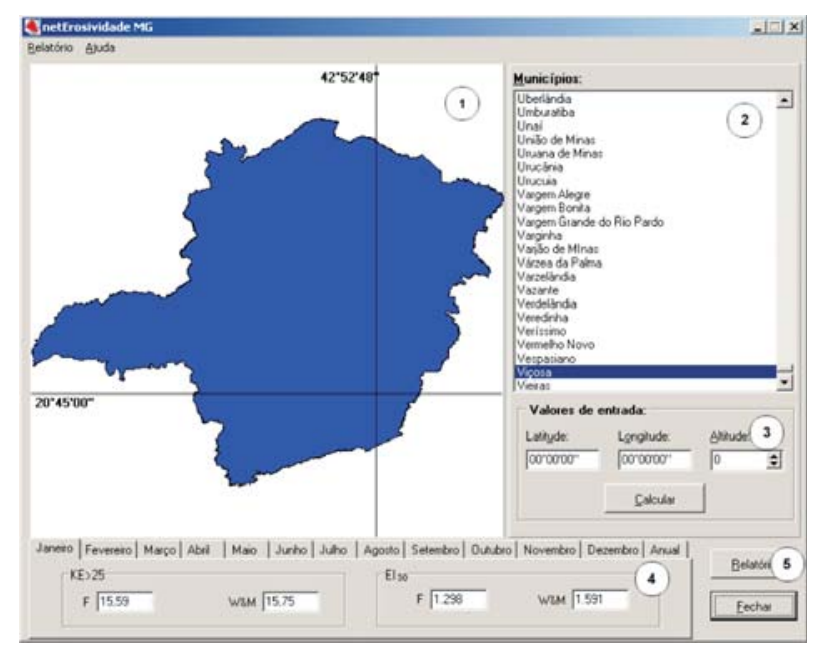

Figura 3. Tela principal do NetErosividade MG.

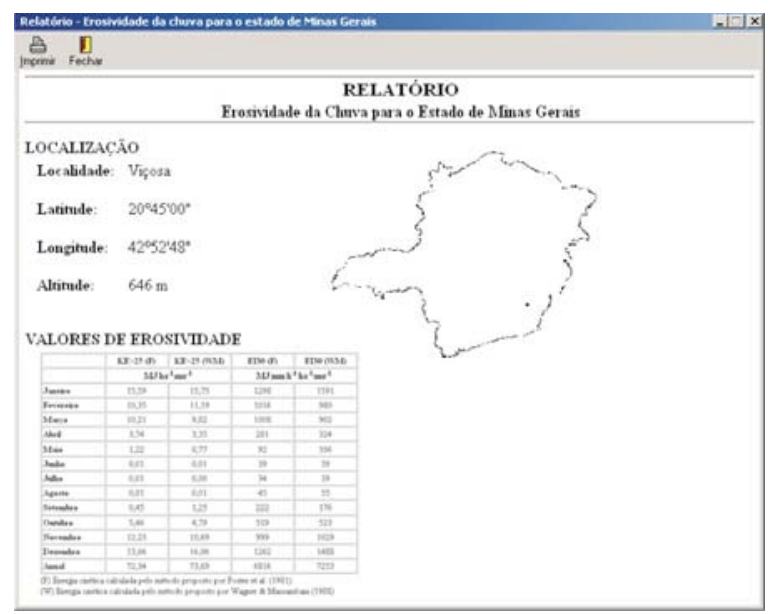

Figura 4. Relatório gerado pelo NetErosividade $M G$ para a localidade de Viçosa. 


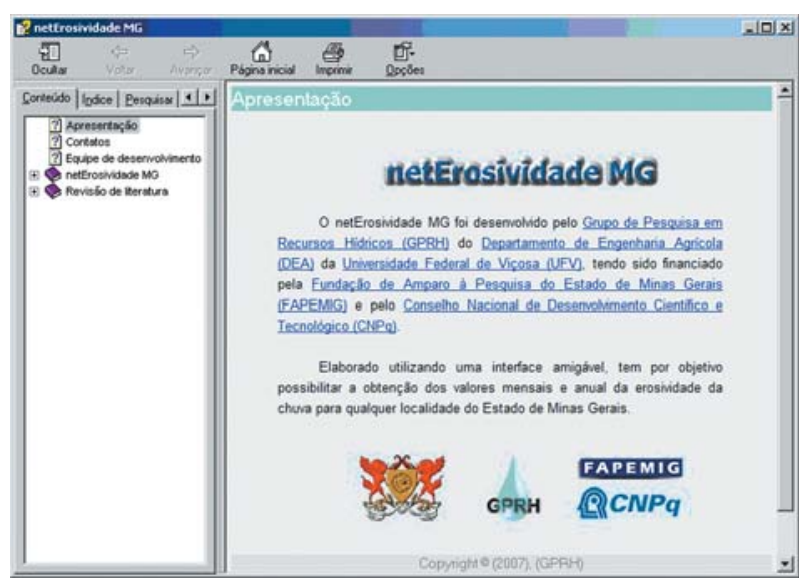

Figura 5. Sistema de ajuda do NetErosividade MG.

\section{CONCLUSÃO}

O NetErosividade $M G$ fornece, de forma fácil e rápida, os valores mensais e anual da erosividade da chuva para qualquer localidade do Estado de Minas Gerais, possibilitando sua utilização por técnicos e extensionistas em atividades que auxiliem na conservação de água e solo.

\section{AGRADECIMENTOS}

Os autores agradecem à Fundação de Amparo à Pesquisa do Estado de Minas Gerais - FAPEMIG e ao Conselho Nacional de Desenvolvimento Científico e Tecnológico - CNPq, pelo apoio financeiro.

\section{LITERATURA CITADA}

AMORIM, R.S.S. Avaliação dos modelos para predição da erosão hídrica USLE, RUSLE e WEPP para condições edafoclimáticas brasileiras. Viçosa, MG, Universidade Federal de Viçosa, 2003. 120p. (Tese de Doutorado)

BERTONI, J. \& LOMBARDI NETO, F. Conservação do solo. 2.ed. São Paulo, Ícone, 1999. 355p.

CAMARGO, A.P. \& SENTElhas, P.C. Avaliação do desempenho de diferentes métodos de estimativa da evapotranspiração potencial no Estado de São Paulo. R. Bras. Agrometeorol., 5:89-97, 1997.

LOMBARDI NETO, F.; PRUSKI, F.F. \& TEIXEIRA, A.F. Sistema para o cálculo da erosividade da chuva para o Estado de São Paulo. Software. Versão 1.0, GPRH / IAC, Viçosa, MG, 1999.

FOSTER, G.R.; McCOOL, D.K.; RENARD, K.G. \& MOLDENHAUER, W.C. Conversion of the universal soil loss equation to SI units. J. Soil Water Conserv., 36:3559, 1981.
MATLAB software. Version 6.0. Natick, The MathWorks, 2000.

MELLO, C.R.; SÁ, M.A.C.; CURI, N.; MELLO, J.M.; VIOLA, M.R. \& SILVA, A.M. Erosividade mensal e anual da chuva no Estado de Minas Gerais. Pesq. Agropec. Bras., 42:537$545,2007$.

MOREIRA, M.C.; CECÍLIO, R.A.; PINTO, F.A.C.; LOMBARDI NETO, F. \& PRUSKI, F.F. Programa computacional para estimativa da erosividade da chuva no Estado de São Paulo utilizando redes neurais artificiais. R. Eng. Agric., 14:8892, 2006a.

MOREIRA, M.C.; CECÍLIO, R.A.; PINTO, F.A.C. \& PRUSKI, F.F. Desenvolvimento e análise de uma rede neural artificial para estimativa da erosividade da chuva para o Estado de São Paulo. R. Bras. Ci. Solo, 30:1069-1074, 2006b.

MOREIRA, M.C.; PRUSKI, F.F.; OLIVEIRA, T.E.C.; PINTO, F.A.C. \& SILVA, D.D. Redes neurais artificiais para estimativa mensal da erosividade da chuva no Estado de Minas Gerais. In: CONGRESSO BRASILEIRO DE ENGENHARIA AGRÍCOLA, 36., Bonito, 2007. Anais. Bonito, Sociedade Brasileira de Engenharia Agrícola, 2007. CD-ROM.

PERSSON, M.; SIVAKUMAR, B.; BERNDTSSON, R.; JACOBSEN, O.H. \& SCHJONNING, P. Predicting the dielectric constant-water content relationship using artificial neural networks. Soil Sci. Soc. Am. J., 66:14241429, 2002.

PRUSKI, F.F.; MOREIRA, M.C.; GONÇALVES, F.A.; OLIVEIRA, T.E.C.; PINTO, F.A.C. \& SILVA, D.D. Erosividade da chuva a partir de séries sintéticas de precipitação. In: CONGRESSO BRASILEIRO DE ENGENHARIA AGRÍCOLA, 36., Bonito, 2007. Anais. Bonito, Sociedade Brasileira de Engenharia Agrícola, 2007. CD-ROM.

RIBEIRO, C.A.A.S.; CHAVES, M.A.; SOARES, V.P. \& EUCLYDES, H.P. Modelos digitais de elevação hidrologicamente consistentes para a Amazônia legal. In: SIMPÓSIO DE RECURSOS HÍDRICOS DO CENTRO OESTE, 2., Campo Grande, 2002. Anais. Campo Grande, Associação Brasileira de Recursos Hídricos, 2002. CDROM

SÁRKÖZY, F. Gis functions - Interpolation. Periodica Polytechnica Ser. Civ. Eng., 43:63-86, 1999.

SILVA, A.M. Rainfall erosivity map for Brazil. Catena, 57:251259,2004

WAGNER, C.S. \& MASSAMBINI, O. Análise da relação intensidade de chuva: Energia de Wischmeier \& Smith e sua aplicabilidade à região de São Paulo. R. Bras. Ci. Solo. 12:197-203. 1988.

WILLMOTT, C.J. On the validation of models. Phys. Geogr., 2:184-194, 1981.

WISCHMEIER, W.H. \& SMITH, D.D. Rainfall energy and its relationship to soil loss. Trans. Am. Geophys Union, 39:285-291, 1958. 